\title{
Reduction of Intrachannel Four-Wave Mixing Using Subchannel Multiplexing
}

\author{
John Zweck and Curtis R. Menyuk, Fellow, IEEE
}

\begin{abstract}
We propose to reduce intrachannel four-wave mixing by using subchannel multiplexing for each wavelength-divisionmultiplexed channel. We calculate the growth in both signal distortion and noise, and we show that there is a tradeoff between propagation distance and spectral efficiency. The method performs best with strong dispersion management and Raman amplification.
\end{abstract}

Index Terms-Nonlinear distortion, optical fiber communication, optical fiber dispersion.

\section{INTRODUCTION}

A MAJOR OBSTACLE system designers must overcome to achieve long-haul propagation in quasi-linear high data rate fiber-optic transmission systems is to reduce pulse distortion due to nonlinear effects. Systems that have a greater tolerance to the fiber nonlinearity can be operated at higher power and hence are also more tolerant to the accumulated effects of noise from erbium-doped or Raman fiber amplifiers. In quasilinear systems, strong dispersion management is used to reduce both interchannel nonlinear effects and pulse distortion due to self-phase modulation. However, in strongly dispersion-managed return-to-zero systems operating at data rates of $40 \mathrm{~Gb} / \mathrm{s}$ and above, the large degree of pulse overlap during transmission results in intrachannel four-wave mixing (FWM) that can limit the propagation distance [1]. In these systems, FWM transfers energy from triples of pump pulses into the bit slots of the spaces, thereby generating ghost pulses. FWM also transfers energy into the bit slots of the marks, inducing jitter in the energy of the marks [2]. When designing new systems, the effects of intrachannel FWM can be reduced by using low-nonlinearity slope-matched fiber, by optimizing the dispersion map, by choosing appropriate amounts of dispersion pre and postcompensation, and by using Raman amplification [3]-[5]. However, intrachannel FWM can also severely limit the propagation distance when installed systems are upgraded to higher per-channel data rates, as for example, when a terrestrial system is upgraded from 10 to $40 \mathrm{~Gb} / \mathrm{s}$. In this situation, it is more cost effective to modify the transmitter and receiver subsystems than to install new fiber or to redesign the dispersion map. Consequently, several novel transmission formats have recently been proposed to decrease intrachannel FWM [6]-[9].

In this letter, we show that the effect of intrachannel FWM can be reduced by replacing each $40-\mathrm{Gb} / \mathrm{s}$ wavelength-divisionmultiplexed (WDM) channel by two $20-\mathrm{Gb} / \mathrm{s}$ subchannels that

Manuscript received August 21, 2002.

The authors are with the Computer Science and Electrical Engineering Department, University of Maryland Baltimore County, Baltimore, MD 21250 USA (e-mail: zweck@umbc.edu).

Digital Object Identifier 10.1109/LPT.2002.806098 are slightly separated in frequency. We note that these two subchannels are not separate channels, which would require them to be separately demultiplexed and detected. Instead, at the receiver the pulses in the two subchannels must not overlap in the time domain. This method works best for systems in which there is substantial pulse overlap during transmission, such as one would encounter when upgrading an installed system from 10 to $40 \mathrm{~Gb} / \mathrm{s}$. The method relies on trading off decreased spectral efficiency for increased propagation distance. To examine this tradeoff, we performed simulations of a single-channel terrestrial system with amplifier noise and a realistic receiver. Using a single carrier, the maximum propagation distance for which the $Q$ factor exceeded 12 was only $800 \mathrm{~km}$. However, by using subchannel multiplexing, where the combined bandwidth of the two subchannels was twice that of the single-carrier signal, the propagation distance was increased to $1600 \mathrm{~km}$.

In a previous letter, we analyzed FWM between pulses in a subchannel-multiplexed system with $D_{+} / D_{-}$fiber [9]. Using noise-free simulations, we found that for a pseudorandom bit sequence there are strong resonances in the mean optical energy in the spaces and in the jitter in the optical energy of the marks, and that away from these resonant subchannel frequency spacings the effect of FWM decreases as the subchannel spacing increases. In this letter, we take noise into account. First, we show that the strength of the resonances is decreased due to the noise. Second, we examine single-mode fiber (SMF)/dispersion compensating fiber (DCF), which is more likely to be encountered in a system upgrade than $D_{+} / D_{-}$fiber, and we show that, in conjunction with hybrid Raman-erbium-doped fiber amplification, the subchannel-multiplexing format can still be useful if increased distance is more important than increased spectral efficiency.

\section{SYSTEM DESCRIPTION}

The subchannel-multiplexed signal is obtained by replacing each WDM channel by a pair of subchannels that are created by shifting the central frequencies of the pulses in the even numbered bit slots by $+\Delta \Omega / 2$ and those in the odd bit slots by $-\Delta \Omega / 2$. For a $40-\mathrm{Gb} / \mathrm{s}$ signal, this procedure produces two $20-\mathrm{Gb} / \mathrm{s}$ signals whose pulse widths are appropriate for a $40-\mathrm{Gb} / \mathrm{s}$ signal and that are spaced $\Delta \Omega$ apart in frequency with a time offset of $25 \mathrm{ps}$. In experiments, subchannel-multiplexed signals could be produced using a hybrid optical time- and WDM scheme. At the receiver, the two subchannels are treated as if they were a single $40-\mathrm{Gb} / \mathrm{s}$ channel. In particular, each pair of subchannels is demultiplexed from the full WDM signal using a single optical filter whose central frequency is the average of the central frequencies of the two subchannels. To 
ensure that at the receiver the pulses in the two subchannels do not overlap in the time domain, we assume that the total accumulated dispersion and dispersion slope are close to zero. We will examine the dispersion tolerance of the subchannel-multiplexing transmission format in Section III.

To understand how subchannel multiplexing reduces FWM, suppose that the envelope of the electric field can be approximated by $u+q$, where $u$ is the solution of the linear dispersive equation and where $q$ is a nonlinear perturbation [10]. The perturbation $q$ is the sum of the perturbations $q_{m, n, l}$ due to nonlinear forcing terms $u_{m} u_{n} \bar{u}_{l}$ in the nonlinear Schrödinger equation, where $u_{k}$ denotes a pump pulse in the $k$ th bit slot. In [9], we showed that, if two of the pump pulses are in one subchannel and the third is in the other subchannel, then the energy of $q_{m, n, l}$ decreases exponentially as $\Delta \Omega$ increases. The reason for this decrease is that the amplitude of the nonlinear forcing function decreases as the pump pulses in the two subchannels move apart during propagation.

We tested the method on a prototypical terrestrial system by performing single-channel simulations using the nonlinear Schrödinger equation. The dispersion map consisted of SMF of length $L=100 \mathrm{~km}$ followed by DCF of length $L / 5$. The SMF had a dispersion of $D_{\mathrm{SMF}}=16 \mathrm{ps} / \mathrm{nm} \cdot \mathrm{km}$ and a dispersion slope of $0.08 \mathrm{ps} / \mathrm{nm}^{2} \cdot \mathrm{km}$ at $1550 \mathrm{~nm}$. The average dispersion and dispersion slope of the map was zero. For the SMF and DCF, the effective areas were 80 and $20 \mu \mathrm{m}^{2}$, respectively, and the nonlinear Kerr coefficient was $2.6 \times 10^{-20} \mathrm{~m}^{2} / \mathrm{W}$. Since intrachannel FWM is reduced by using an accumulated dispersion function that is approximately symmetric about the zero-dispersion axis [5], we used linear dispersion precompensation of $-D_{\mathrm{SMF}} L / 2$ and linear postcompensation of $+D_{\mathrm{SMF}} L / 2$. Using the method described above, we obtained the input subchannel-multiplexed signal from a $40-\mathrm{Gb} / \mathrm{s}$ return-to-zero pulse train that used a pseudorandom bit sequence of length $2^{10}$ and consisted of raised-cosine shaped pulses with a central frequency of $1550 \mathrm{~nm}$. For this system, the maximum pulse duration during propagation is 15 -b slots and, when $\Delta \Omega=75 \mathrm{GHz}$, the maximum walkoff between pulses in the two subchannels is 19-b slots. The receiver consisted of a single $200-\mathrm{GHz}$ Gaussian optical filter with a central wavelength of $1550 \mathrm{~nm}$, a square-law photodetector, and a $35-\mathrm{GHz}$ fourth-order electrical Bessel filter. The $Q$ factor was computed using the standard formula $Q=\left(\left\langle i_{1}\right\rangle-\left\langle i_{0}\right\rangle\right) /\left(\sigma_{1}+\sigma_{0}\right)$ by averaging over $2^{10} \mathrm{~b}$.

A backward pumped fiber Raman amplifier compensated for the loss in the SMF. This amplifier was modeled using a continuous-wave (CW) approximation without pump depletion. The loss in the SMF was $0.25 \mathrm{~dB} / \mathrm{km}$ at the pump wavelength of $1450 \mathrm{~nm}$ and $0.2 \mathrm{~dB} / \mathrm{km}$ at the signal wavelength of $1550 \mathrm{~nm}$. The ON-OFF Raman gain was $G_{\mathrm{ON}-\mathrm{OFF}}=20 \mathrm{~dB}$. The noise due to the Raman amplifier was added immediately after the SMF by attenuating the signal by $G_{\mathrm{ON}-\mathrm{OFF}}$, and then restoring the power using an erbium-doped fiber amplifier (EDFA) with a noise figure of $\mathrm{NF}_{\text {eff }}=-0.8 \mathrm{~dB}$. This noise figure is the experimentally measured effective noise figure for a backward pumped Raman amplifier in SMF with $G_{\mathrm{ON}-\mathrm{OFF}}=20 \mathrm{~dB}$ [11]. An EDFA, with a noise figure of $3.8 \mathrm{~dB}$ that was placed after the DCF, compensated for the loss of $0.5 \mathrm{~dB} / \mathrm{km}$ in the DCF.

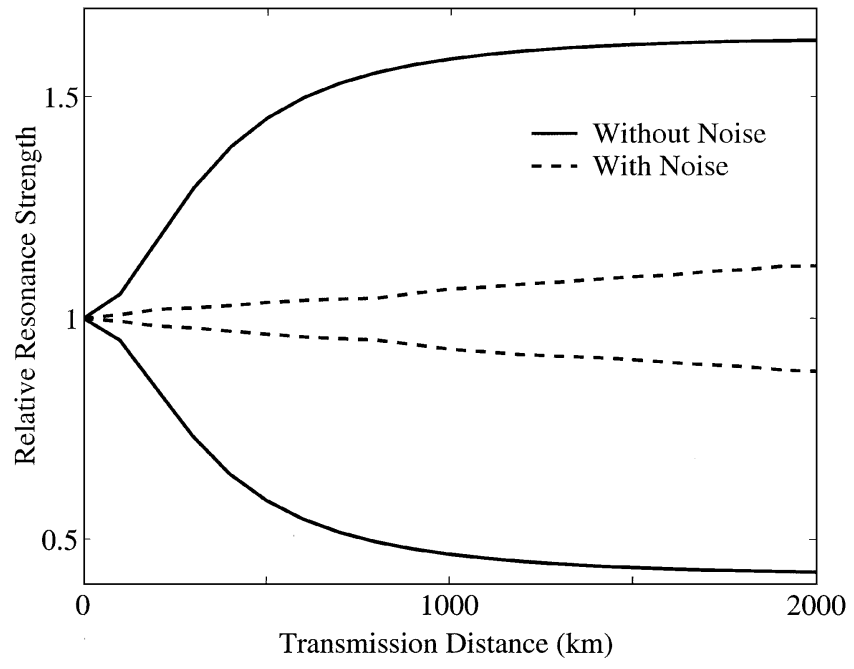

Fig. 1. The optima with respect to $\Delta \phi$ of the mean received voltage in the spaces, relative to its $\Delta \phi$ average, versus transmission distance when $\Delta \Omega=0$.

\section{RESULTS}

In our first simulation study, we examined how the strength of the intrachannel FWM resonances depends on the noise from the optical amplifiers. Since FWM is a coherent process, we introduced a constant phase difference $\Delta \phi$ between the pulses in the odd and in the even numbered bit slots. We found that there are resonances in the mean of the received voltage in the spaces and in the standard deviation of the received voltage in the marks as functions of the subchannel frequency spacing $\Delta \Omega$, and that the strength of these resonances depends on the phase difference $\Delta \phi$. In Fig. 1 , for $\Delta \Omega=0$, we plot the strength of the resonance of the mean voltage in the spaces as a function of the transmission distance through the SMF. We performed simulations with and without amplifier noise. The two solid curves are the maximum and the minimum of this mean voltage in the case of noise-free simulations, where the optima are taken with respect to $\Delta \phi$. At each distance, the voltages are given relative to the phase-averaged mean voltage at that distance. The relative strength of the resonance is given by the difference between the two curves. The two dashed curves show the corresponding results for simulations with noise. This result demonstrates that noise disrupts the growth in the strength of the FWM resonances. The reason the resonances are weaker in the presence of noise is that the noise randomizes the phases of the pump pulses, and hence of the nonlinear perturbations that contribute energy into the different bit slots. We observed similar qualitative behavior for the standard deviation of the voltage in the marks. The degree to which the noise disrupts the growth of the resonances depends on the peak power of the signal and on the signal-to-noise ratio (SNR). For the results shown in Fig. 1, the peak power was $2 \mathrm{~mW}$, and the SNR was $18 \mathrm{~dB}$ after $2000 \mathrm{~km}$ of propagation through the SMF. The resonances are stronger for higher peak powers and lower SNRs.

In our second simulation study, we examined the tradeoff between increasing propagation distance and decreasing spectral efficiency for the subchannel-multiplexing transmission format. In Fig. 2, we plot the maximum propagation distance over which $Q>12$ for the subchannel-multiplexed signal 


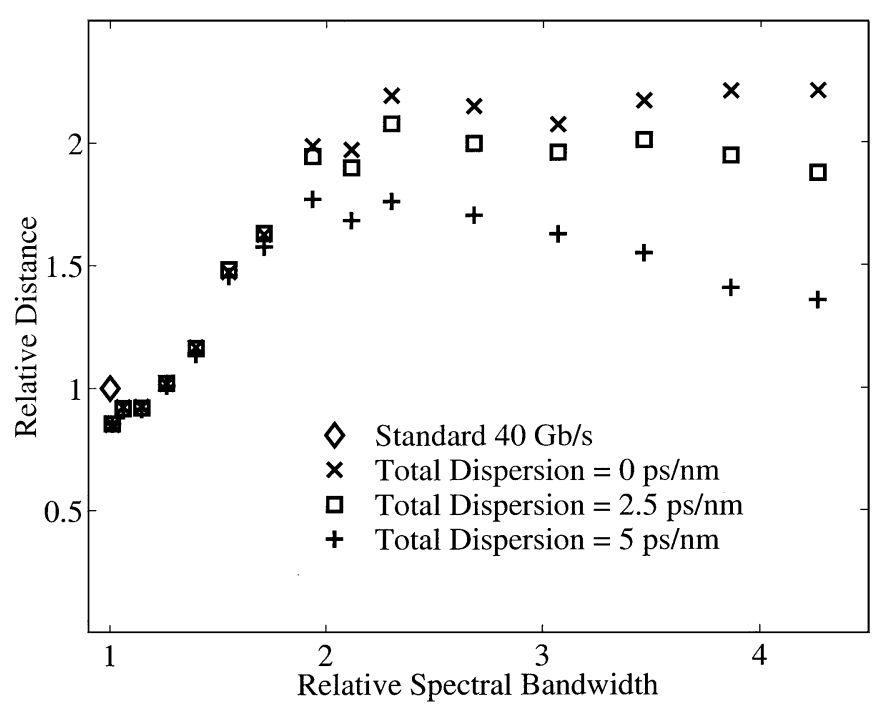

Fig. 2. Distance for which $Q>12$ versus signal bandwidth for a subchannel-multiplexed signal relative to a standard $40-\mathrm{Gb} / \mathrm{s}$ signal.

as a function of the spectral bandwidth. All distances and bandwidths in Fig. 2 are given relative to the corresponding quantities for a standard 40-Gb/s signal with zero total accumulated dispersion at the receiver, $D_{\text {tot }}=0$. By a standard $40-\mathrm{Gb} / \mathrm{s}$ signal, we mean a $40-\mathrm{Gb} / \mathrm{s}$ signal with $\Delta \Omega=0$ and $\Delta \phi=0$. We used the rms spectral width as a measure of the signal bandwidth. We chose the subchannel frequency spacings $\Delta \Omega$ to avoid the resonances in the spaces and marks, and for each $\Delta \Omega$ we optimized the peak power of the transmitted signal and chose $\Delta \phi$ to be zero. The subchannel frequency spacings in gigahertz and the peak power levels in milliwatts were $\left(\Delta \Omega, P_{0}\right)=(7,2),(17,4),(27,4),(37,4),(47,4),(57,4),(67,4)$, $(80,6),(90,6),(100,8),(120,8),(140,8),(160,8),(180,8)$, and $(200,8)$. For these subchannel spacings, the results are insensitive to the choice of $\Delta \phi$. As $\Delta \Omega$ increases the degradation due to FWM decreases, and the tradeoff between the need for low nonlinearity and the need for a high SNR shifts in favor of higher peak powers. For the results that we show with crosses, $D_{\text {tot }}=0$. The result for the standard $40-\mathrm{Gb} / \mathrm{s}$ signal, that we show with a diamond, corresponds to a propagation distance of $800 \mathrm{~km}$ and a spectral bandwidth of $24 \mathrm{GHz}$. When the subchannel frequency spacing is $\Delta \Omega=80 \mathrm{GHz}$ and the relative spectral bandwidth is two, we can propagate almost twice as far as for the standard $40-\mathrm{Gb} / \mathrm{s}$ signal. To examine the dispersion tolerance of the subchannel-multiplexed format, we studied the performance when $D_{\text {tot }}$ was nonzero. For these simulations we added a short piece of linear fiber immediately before the receiver. The squares and plus signs show the results for $D_{\text {tot }}=2.5$ and $5 \mathrm{ps} / \mathrm{nm}$, respectively. The dispersion tolerance decreases as $\Delta \Omega$ increases and becomes a significant factor when $\Delta \Omega \geq 80 \mathrm{GHz}$.

\section{CONCLUSION}

We have proposed to decrease the effects of intrachannel FWM in high data-rate systems by replacing each channel with two subchannel-multiplexed channels. This method trades off spectral efficiency for propagation distance, and it performs best for systems in which there is a large amount of pulse overlap. Consequently, the method may be useful when installed systems are upgraded to higher per-channel data rates.

\section{REFERENCES}

[1] R.-J. Essiambre, B. Mikkelsen, and G. Raybon, "Intra-channel crossphase modulation and four-wave mixing in high-speed TDM systems," Electron. Lett., vol. 35, no. 18, pp. 1576-1578, 1999.

[2] P. V. Mamyshev and N. A. Mamysheva, "Pulse-overlapped dispersionmanaged data transmission and intrachannel four-wave mixing," Opt. Lett., vol. 24, no. 21, pp. 1454-1456, 1999.

[3] A. Mecozzi, C. B. Clausen, and M. Shtaif, "System impact of intrachannel nonlinear effects in highly dispersed optical pulse transmission," IEEE Photon. Technol. Lett., vol. 12, pp. 1633-1635, Dec. 2000.

[4] A. Mecozzi, C. B. Clausen, M. Shtaif, S.-G. Park, and A. H. Gnauck, "Cancellation of timing and amplitude jitter in symmetric links using highly dispersed pulses," IEEE Photon. Technol. Lett., vol. 13, pp. 445-447, May 2001.

[5] P. Johannisson, D. Anderson, A. Berntson, and J. Martensson, "Generation and dynamics of ghost pulses in strongly dispersion-managed fiber-optic communication systems," Opt. Lett., vol. 26, no. 16, pp. 1227-1229, 2001

[6] S. Kumar, "Intrachannel four-wave mixing in dispersion managed RZ systems," IEEE Photon. Technol. Lett., vol. 13, pp. 800-802, Aug. 2001.

[7] K. S. Cheng and J. Conradi, "Reduction of pulse-to-pulse interaction using alternative RZ formats in 40-Gb/s system," IEEE Photon. Technol. Lett., vol. 14, pp. 98-100, Jan. 2002.

[8] X. Liu, X. Wei, A. H. Gnauck, C. Xu, and L. K. Wickham, "Suppression of intrachannel four-wave-mixing-induced ghost pulses in high-speed transmission by phase inversion between adjacent marker blocks," Opt. Lett., vol. 27, no. 13, pp. 1177-1179, 2002.

[9] J. Zweck and C. R. Menyuk, "Analysis of four-wave mixing between pulses in high-data-rate quasilinear subchannel-multiplexed systems," Opt. Lett., vol. 27, no. 14, pp. 1235-1237, 2002.

[10] A. Mecozzi, C. B. Clausen, and M. Shtaif, "Analysis of intrachannel nonlinear effects in highly dispersed optical pulse transmission," IEEE Photon. Technol. Lett., vol. 12, pp. 392-394, Apr. 2000.

[11] P. B. Hansen, L. Eskildsen, J. Stentz, T. A. Strasser, J. Judkins, J. J. DeMarco, R. Pedrazzani, and D. J. DiGiovanni, "Rayleigh scattering limitations in distributed Raman pre-amplifiers," IEEE Photon. Technol. Lett., vol. 10, pp. 159-161, Jan. 1998. 